\title{
Le recrutement de patients dans le monde numérique occupé d'aujourd'hui
}

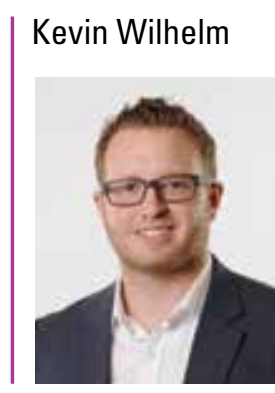

Kevin Wilhelm est président de 4ECP, entreprise de ressources professionnelles pour les professionnels des soins oculovisuels. $4 E C P$ s offre des services portant sur l'emploi, la formation et le marketing. Pour en savoir davantage, consultez son site Web à www.4ecps.com ou envoyez un message électronique à kevin@4ecps.com.
$\mathrm{L}$ e recrutement de nouveaux patients constitue un des plus gros défis que doit relever le professionnel indépendant des soins oculovisuels dans le monde numérique d'aujourd'hui. Chaque entreprise de chaque secteur a besoin de nouveaux clients afin de survivre, mais le paysage a changé de façon spectaculaire depuis une décennie. L'environnement concurrentiel dépasse les chaînes de grandes surfaces et englobe maintenant les détaillants en ligne, financés par des investisseurs en capital de risque et qui cherchent à occuper la plus grande part de marché possible. Pour demeurer viable, il faut respecter un ensemble de règles en particulier - établies pour assurer la solidité de votre entreprise de soins oculovisuels.

\section{Règle 1 - Être prêt à jouer le jeu}

L'époque où l'on pouvait diriger une grande entreprise et s'attendre à une croissance d'origine interne basée sur les références est révolue. Toutes les entreprises obtiendront des références, mais pas suffisamment pour en survivre. Les détaillants en ligne sont devant tous les clients tous les jours. Ils savent comment les cibler, ce qu'il faut dire et ils offrent la commodité de commander en ligne. Pour affronter les détaillants en ligne, vous devez vous retrouver sur le même terrain qu'eux - en ligne! Si les détaillants en ligne investissent dans le marketing sur Facebook, il faut le faire vous aussi. Pourquoi pas - si cela ne marchait pas pour eux, ils ne continueraient pas d'investir énormément dans le marketing en ligne.

Il y a deux façons de faire vraiment grossir votre cabinet de soins oculovisuels dans le monde numérique d'aujourd'hui - un excellent point de vente au détail (et oui, ça marche toujours!) ou le marketing en ligne. Il n'y a pas vraiment moyen d'y échapper. Les gens sont occupés, distraits, impatients et sensibles aux prix. Pour attirer leur attention (et leur portefeuille), il faut vous trouver au bon endroit au bon moment. Il faut à cette fin soit investir dans un endroit très fréquenté par les piétons, soit élargir votre empreinte en ligne. Combien faut il investir? Voilà une excellente question, mais il existe malheureusement trop de variables pour présenter une réponse universelle. Le bon montant dépend des buts fixés par votre entreprise et de la combinaison de facteurs internes et externes auxquels elle fait face. Il faut en général être prêt à investir de $10 \%$ à $15 \%$ de la croissance recherchée. Par exemple, si vous voulez augmenter votre chiffre d'affaires de $200000 \$-$ il faudrait être prêt à investir au moins 20000 \$ à $25000 \$$ dans le marketing de votre entreprise en ligne.

\section{Règle 2 - Trouver votre argument de vente}

Les détaillants en ligne ont leur argument de vente. Ils offrent des prix plus bas et une plus grande commodité en permettant à leurs patients de commander de n'importe où et n'importe quand. Nous connaissons tous les inconvénients toutefois les retours causent des ennuis, le choix peut être limité, sans un opticien agréé, le bon produit est difficile à trouver pour le patient et les gens ne savent pas de quoi ils auront l'air avec leur nouvelle monture ou leurs lunette fumées.

Les avantages semblent l'emporter sur les inconvénients pour la plupart des consommateurs et la balance continue de pencher en leur faveur. Les détaillants indépendants doivent faire connaître leur argument de vente, raison pour laquelle il faut les choisir - leur raison d'inciter quelqu'un à se lever et à prendre son auto pour aller acheter de nouvelles lunettes 
au lieu de les faire livrer chez soi. Donc... quel est votre argument de vente?

Je vous en prie, ne répondez pas en disant « C'est mon excellent service à la clientèle ». Ce n'est pas le cas. L'excellence du service est un incontournable pour toute entreprise qui veut survivre maintenant : ce n'est pas une proposition de valeur. Sans un excellent service, votre entreprise n'existerait pas. Qu'est ce que vous offrez donc d'autre qui indique que votre entreprise est spéciale? Offrez-vous des marques exclusives, utilisez-vous une technologie à la fine pointe, offrez-vous un vaste éventail de produits ou des prix moins élevés que partout ailleurs? Votre avantage concurrentiel doit l'emporter sur celui des entreprises qui vous entourent - vous devez savoir ce que les détaillants en ligne offrent.

\section{Règle 3 - La promotion de votre argument de vente}

Après avoir choisi votre argument de vente, il faut en faire la promotion efficace auprès de votre clientèle. Le fait d'offrir une gamme de montures à la mode très recherchées vous aidera seulement si vos patients savent que vous l'offrez. Investir dans une nouvelle technologie qui détecte plus rapidement les maladies oculaires, c'est excellent pour votre entreprise, mais l'investissement vous offre une valeur limitée sauf si les gens viennent profiter spécifiquement de la technologie en cause. La promotion peut inclure des publicités sur les canaux numériques ou le vidéomarketing. Elle peut aussi inclure une campagne de bouche à oreille. Il faut absolument informer votre équipe interne au sujet de la façon de positionner votre proposition de valeur auprès de chaque client qui franchit le seuil de votre porte.

Afin de faire concurrence aux détaillants en ligne dans notre monde numérique occupé, il faut chercher avant tout à offrir une expérience impossible à trouver en ligne en déterminant votre argument de vente et vous assurant que le monde le connaît. 